\title{
HERRAMIENTAS FINANCIERAS PARA PYMES EN CONTEXTOS DE INCERTIDUMBRE. ACERCAMIENTO A SU APLICACION
}

Padován, Alex A.

\section{RESUMEN:}

La economía argentina se caracteriza por sucesivos periodos de inestabilidad marcada en las principales variables macroeconómicas lo que suscita la generación de un contexto volátil donde la incertidumbre enmarca cualquier toma de decisión económica y financiera. Este contexto es particularmente nocivo para los negocios de las empresas pequeñas y medianas, quienes encuentran serios inconvenientes para lograr rentabilidad económica del negocio, generando a posteriori estrangulamientos en el giro financiero, todo lo cual puede afectar la capacidad de la PYME para continuar operativa durante estos ciclos económicos. Este trabajo de investigación tiene como objetivo plantear el abordaje de determinadas herramientas que pueden permitir, en la actualidad morigerar el impacto sobre la rentabilidad financiera del negocio y tener la posibilidad de transitar el ciclo económico adverso con éxito.

PALABRAS CLAVES: Incertidumbre, Variables Macroeconómicas, Herramientas

Financieras, Empresas Pymes

\section{ABSTRACT:}

Argentine economy is characterized by successive periods of marked inestability in its key macroeconomic variables, which implies a volatile context where uncertainty frames any economic and financial decision-making. This context is particularly harmful to small and medium-sized companies, who find serious inconveniences to achieve economic profitability of their business, subsequently generating bottlenecks in financial parameters, all of which may affect its ability of remain operative during these cycles.

The aim of this research paper is approaching certain tools that should allow to moderate the impact on business' financial profitability and reach the possibility of successfully going through adverse economic cycle.

KEYWORDS: Uncertainty, Macroeconomic Variables, Financial Tools, SMEs 


\section{INTRODUCCION}

Si hubiera que describir el escenario de la economía argentina con un solo adjetivo, muy posiblemente habría consenso en una palabra: volátil. Los vaivenes económicos y políticos que caracterizan el desarrollo de nuestro país, presentan un contexto complejo y sumamente desafiante para los agentes económicos que operan e interactúan en él, tanto empresas como particulares, suponiendo un severo condicionante para su desarrollo sustentable a mediano y largo plazo.

En el presente artículo, me focalizaré en analizar las características que definen a un escenario de incertidumbre desde el punto de vista económico y de sus principales variables macro y cómo influyen en el desenvolvimiento operativo y financiero de las Pequeñas y Medianas Empresas (en adelante "Pymes"1), motor fundamental de nuestra economía e intentaré presentar ciertas herramientas financieras cuyo conocimiento, entendimiento y utilización se tornan indispensables para lograr una exitosa gestión económico-financiera.

La elección del enfoque de estudio en las Pymes no es azarosa, sino que responde a una realidad en cuanto a la importancia de las mismas para el andamiaje económico de nuestro país, aportando significativamente a la generación de valor, empleo y riqueza.

\section{ESCENARIO ECONOMICO INCIERTO: SUS CARACTERISTICAS}

La economía argentina se ha caracterizado históricamente por la volatilidad y fragilidad de sus principales variables macroeconómicas, incluyendo dentro de dicho conjunto a la variación del Producto Bruto Interno, el Tipo de Cambio, la Tasa de Inflación y la Tasa de Interés de referencia.

Esta situación genera un contexto inestable y sumamente desafiante para el crecimiento de las Pymes en nuestro país, en muchos casos, impidiendo su desarrollo y deteriorando fuertemente la capacidad de generación de rentabilidad y creación de valor.

Analizando de manera paulatina cada una de las variables arriba mencionadas y su evolución y situación actual, permite ir comprendiendo el efectivo nocivo que generan en la consecución de un crecimiento sostenible de la economía Pyme.

En primer lugar, con respecto a la variación del Producto bruto interno (PBI), la Argentina se caracteriza por sus vaivenes y ciclos con mucha dispersión en el comportamiento del PBI, recorriendo períodos de crecimiento a "tasas chinas" como podríamos definir a los años 2003 a 2007 y etapas de estancamiento o recesión, redundando en fuertes caídas de la

\footnotetext{
${ }^{1}$ En Argentina, se consideran Pymes aquellas empresas que se ubiquen dentro de determinados parámetros de Facturación y cantidad de empleados, determinados para las siguientes categorías: Agro, Comercio, Construcción, Industria y Minería y Servicios, mediante Resolución № 69/2020 del Ministerio de Desarrollo Productivo de la Nación.
} 
actividad económica, situación que se observa desde 2008 al presente. Esto plantea para las Pymes la imposibilidad de estimar razonablemente la demanda futura sobre sus productos y servicios, dificultando la construcción de resultados económicos y financieros proyectados, todo lo cual lleva al empresario Pyme, en muchas ocasiones, a decidir la ejecución de sus proyectos de inversión basándose en el factor "intuición", lo cual se traduce a posteriori en situaciones de estrangulamiento financiero producto del descalce de fondos generados por la decisión y en la consecuente destrucción de valor para la Pyme, hecho que, a todas luces, se evitaría con una correcta y oportuna formulación y evaluación del proyecto involucrado en la decisión. Veremos en el próximo apartado de este trabajo como utilizar el Análisis de Escenarios para intentar resolver este limitante.

En segundo lugar, respecto al comportamiento del Tipo de Cambio, claramente encontramos aquí otra variable cuya evolución impacta notoriamente en el escenario económico para las Pymes. Nuestro país no ha logrado todavía contar con una moneda de curso legal que sea visualizada por los agentes económicos como resguardo de valor y ello genera constantes presiones sobre el tipo de cambio, lo cual para un país con inconvenientes estructurales para generar ingresos de divisas presupone la generación de crisis cambiarias que, históricamente han culminado con procesos devaluatorios de la moneda local. Esto plantea para las Pymes una dificultad importante para poder analizar y definir inversiones en Bienes de capital o insumos dolarizados, a raíz de no poder estimar con certeza el importe en pesos que deberán afrontar oportunamente.

Veremos en el próximo apartado de este trabajo como utilizar el Mercado de Futuros de Dólar y la Teoría de Paridad de Tasas de Interés para intentar resolver este limitante.

En tercer lugar, respecto a la Tasa de Inflación, encontramos en este punto, otra variable macroeconómica cuyo comportamiento influye negativamente en el desempeño económico y financiero e la Pyme, sobre todo en cuanto a la compresión de sus márgenes de rentabilidad. Es honesto mencionar que nuestro país convive desde hace más de una década con niveles de inflación superiores al 20/25\%, lo que conlleva un deterioro del poder adquisitivo de la moneda local y por añadidura, una distorsión de los precios relativos de la economía. Esto afecta a la Pyme producto del incremento sobre sus costos fijos, situación que redunda en una desmejora de sus márgenes bruto y operativo, dado que no siempre pueden trasladar a precios dichos incrementos. Presentaremos en el próximo apartado las herramientas Leverage Operativo y Leverage Financiero que ayudarán a la empresa a medir el potencial deterioro de sus márgenes.

En último lugar, debemos mencionar a la variable del nivel de Tasa de Interés de referencia de la economía. Claramente nuestro país no cuenta con un mercado bancario y de capitales profundo, lo cual repercute en la escasez de financiamiento para las pymes a la hora de analizar alterativas para cubrir los requerimientos de inversión de sus proyectos, máxime en 
un contexto donde el nivel de tasas de interés es una variable de política monetaria a través de la cual el Banco Central de la República Argentina busca contener las demandas inflacionarias y cambiarias. Esto ha generado un encarecimiento del crédito y un corrimiento del sector privado de la oferta de crédito, repercutiendo en el costo del financiamiento para la Pyme, que en muchas ocasiones se torna antieconómico y produce una situación de desventaja financiera, destruyendo valor para la misma. Introduciremos en el apartado siguiente la herramienta de Leverage de Balance General, que será de utilidad para medir la conveniencia o no de convalidar e incorporar un préstamo a la estructura patrimonial del negocio.

\section{HERRAMIENTAS FINANCIERAS DE UTILIDAD PARA LA PYME}

En el apartado anterior, se han recorrido las principales variables macroeconómicas, analizando su condición en nuestro país y el impacto que genera en las Pymes la volatilidad en su comportamiento.

En este capítulo, introduciremos al lector algunas herramientas financieras que permitirán morigerar (o en algún caso, eliminar) el riesgo subyacente al operar en contextos de incertidumbre.

En primera medida, es menester abordar el Análisis de Escenarios como herramienta para atenuar el efecto que suscita la volatilidad del PBI en las proyecciones económicas de la empresa. Según Lopez Dumrauf, los cambios en las variables macroeconómicas producen modificaciones en los indicadores económicos y en el flujo de fondos de las empresas, por lo que es indispensable a la hora de construir presupuestos y proyecciones que la Pyme sensibilice las mismas bajo el marco de un esquema de Escenarios Ponderados, esto es, determinar las características, el comportamiento y el valor que se estiman tendrán a futuro las principales variables económicas y asignarles una ponderación en base a su probabilidad de ocurrencia. De esta manera, las proyecciones que realice la Pyme no quedarán enteramente condicionadas y sustentadas en un único contexto a futuro sino que estarán en condiciones de absorber los cambios que se puedan suscitar, buscando siempre ponderar en mayor medida escenarios desfavorables.

En segunda medida, se presenta a continuación otra herramienta de vital importancia para la Pyme a los fines de mitigar y, puntualmente, en esta cuestión eliminar el riesgo cambiario. Es notorio como el deterioro de la moneda local frente a monedas extranjeras, principalmente el Dólar de Estados Unidos, repercute negativamente en las inversiones de Bienes de Capital que las Pymes precisan para sostener o mejorar su capacidad productiva, en virtud de no poder presupuestar con certeza que valor deberán afrontar en moneda local para dicha inversión. 
Los contratos de Dólar Futuro que se negocian en el Mercado de Futuros de Rosario y Buenos Aires (en adelante Matba/Rofex) ${ }^{1}$ y permiten a los participantes realizar operaciones de coberturas sobre sus flujos de fondos a partir de la compra o venta de dichos contratos en las distintas posiciones ofrecidas. Como características generales, se puede mencionar que se trata de contratos estandarizados, en donde cada uno equivale a USD 1.000 y se negocian para cada uno de los próximos doce meses calendario, resolviéndose por liquidación de diferencias. Por lo tanto, una Pyme que precise eliminar el riesgo cambiario sobre su flujo de fondos, producto por ejemplo, de la concertación de una operación de importación puede acceder a este mercado a cubrir la cantidad de dólares que requiere disponer y de esa manera, otorgar certeza a sus proyecciones financieras.

Adicionalmente, en situaciones donde la Pyme deba incorporar el tipo de cambio dentro de sus modelos financieros, se puede recurrir a la Teoría de Paridad de Tasas de Interés, por la cual, como establece Van Horne se puede estimar el tipo de cambio futuro de una moneda respecto a otra, tomando el tipo de cambio actual multiplicado por el rendimiento marginal de un bono soberano en pesos por sobre un bono soberano en dólares, a saber: TC futuro $=$ TC actual $x(1+i \$) /(1+i U S D)$.

En tercera medida, se explicó en el apartado anterior el efecto negativo sobre los márgenes bruto, operativo y neto que provocan los escenarios inflacionarios recurrentes de nuestro país. Por consecuente, es oportuno presentar en este punto dos herramientas útiles para evaluar el resultado que tendría sobre el Resultado Operativo y el Resultado Neto, variaciones en el nivel de ventas de la Pyme: Leverage Operativo y Leverage Financiero.

Lopez Dumrauf define al Leverage Operativo como una herramienta de sensibilidad que muestra cómo cambia el resultado operativo cuando se producen cambios en el nivel de ventas representando el "efecto palanca" sobre los resultados operativos, como consecuencia de la existencia de costos fijos. Matemáticamente se puede obtener de la siguiente manera: $L O P=$ Contribución marginal / Resultado Operativo. De esta forma, utilizando esta herramienta la Pyme puede conocer el impacto probable en su margen operativo como consecuencia de una variación en la facturación, por ejemplo, por adecuación de lista de precios por inflación. Paralelamente, el mismo autor define al Leverage Financiero como la herramienta que muestra como cambia el resultado neto (o rentabilidad del capital propio) cuando se utiliza financiamiento ajeno, representando, en este caso, el "efecto palanca" sobre el resultado neto, como consecuencia de la existencia de costos fijos por intereses financieros. Matemáticamente se obtiene de ls siguiente manera: $L F=$ Resultado Operativo/(Resultado Operativo-Intereses).

\footnotetext{
${ }^{1}$ Fundado en 2019, Matba Rofex nace de la fusión de dos mercados de tradición centenaria en la operatoria de futuros y opciones en Argentina, el Mercado a Término de Buenos Aires S.A. y ROFEX S.A.
} 
Por lo expuesto anteriormente, la combinación de ambas herramientas permite a la Pyme estimar como responderían sus márgenes operativo y neto ante modificaciones en el volumen de facturación.

En última medida, se abordó en el apartado anterior el problema de la tasa de interés y como el creciente comportamiento de la misma en nuestro país relega a las pymes de la posibilidad de acceder a financiamiento, en calidad y cantidad, para sus proyectos de inversión. En este punto, se presenta una herramienta interesante para medir la calidad del financiamiento que toma la Pyme: el Leverage de Balance General (LBG). Conceptualmente esta herramienta indica el cuanto cambia la rentabilidad financiera (o Rentabilidad del capital propio) en virtud de una nueva toma de deuda, con respecto a una situación previa sin endeudamiento, manteniendo constante el resultado operativo.

Desde una óptica matemática, el LBG puede obtenerse a partir de:

$\angle B G=(($ Resultado Operativo -Intereses)/ Resultado Operativo) $\times$ (Activo/PN);

Es interesante entender la herramienta desde una perspectiva gráfica, a saber:

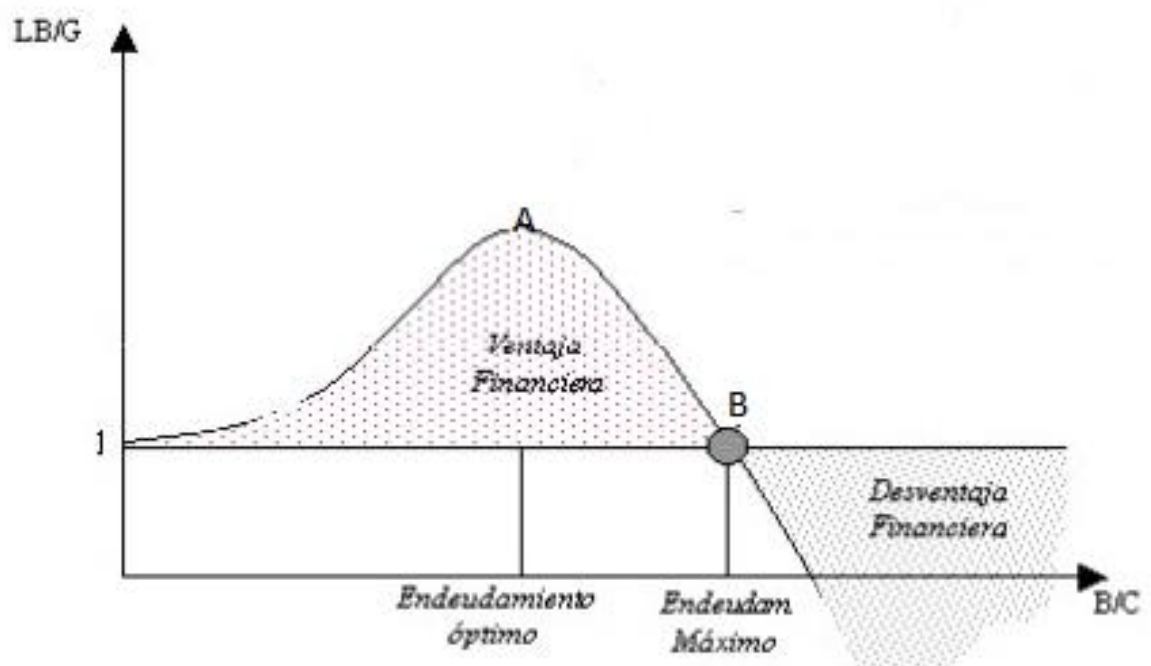

Del análisis gráfico, puede entenderse que, en un primer momento, a medida que la Pyme va incorporando deuda a su estructura patrimonial, se va desarrollando una situación de "Ventaja financiera", por la cual el rendimiento operativo que genera el nuevo financiamiento supera su costo, teniendo como punto máximo (punto A) el punto de endeudamiento óptimo, en el cual la ventaja financiera se amplifica. Sin embargo, a medida que la Pyme continúa incrementando su nivel de endeudamiento, esa ventaja comienza a evaporarse (punto B endeudamiento máximo) y se transforma en una situación de Desventaja Financiera, por la cual el costo de la deuda supera el rendimiento operativo que la Pyme logra con su incorporación. En síntesis, esta herramienta permite medir la conveniencia o no, en términos de generación de valor para la Pyme, de una decisión de financiamiento con deuda. 


\section{CONCLUSIONES}

A lo largo de este trabajo, se ha intentado presentar y explicar de manera sucinta, distintas herramientas disponibles para los empresarios Pyme tendientes a morigerar e hecho de desarrollar su actividad en un contexto incierto y volátil como el que presenta nuestra realidad local. La intención es que lo vertido en estas líneas permita ser usado como disparador para la incorporación de estas herramientas en los tableros de comando y procesos decisorios que sean encarados de aquí en adelante.

\section{REFERENCIAS BIBLIOGRAFICAS}

Allen, F.;Brealey, R. y Myers, S. (2011). Principles of Corporate Finance. Estados Unidos. Editorial Mc-Graw Hill.

Lopez Dumrauf, G. (2010). Finanzas corporativas. Un enfoque latinoamericano. Argentina. Editorial Alfaomega.

Van Horne, J. (2000). Administración Financiera. 10ª Edición.; México. Editorial Prentice Hall.

Matba-Rofex. Productos ofrecidos: Contratos de USD futuros. Disponible en: https://www.matbarofex.com.ar/producto/futuros-y-opciones-sobre-dolar

Ministerio de Desarrollo Productivo de la Nación. Resolución $N^{\circ}$ 69/2020. Disponible en Boletín Oficial de la República Argentina:

https://www.boletinoficial.gob.ar/detalleAviso/primera/231104/20200624 